\title{
Proceso de gestión del conocimiento. Experiencia en la industria petrolera venezolana
}

\author{
Knowledge management process. Experience in Venezuelan oil \\ industry
}

\section{Norbellys Coromoto Urribarri Estrada \\ norbelisurribarri@gmail.com \\ ORCID 0000-0001-9277-705X \\ Universidad del Zulia, Venezuela}

Artículo recibido febrero 2020 | Arbitrado en marzo 2020 | Publicado en mayo 2020

\section{RESUMEN}

El propósito de la investigación fue describir el proceso de gestión del conocimiento en las gerencias de operaciones acuáticas de la industria petrolera en el estado Zulia. El tipo de investigación fue descriptiva, de campo, transeccional no experimental. La población estuvo conformada por 46 sujetos entre líderes, gerentes y supervisores. Se utilizó la encuesta, mediante un cuestionario, validado por el juicio de expertos. La confiabilidad se determinó mediante el Coeficiente de Alpha Cronbach, cuyo valor fue 0,95 (muy alta confiabilidad). Se aplicó la estadística descriptiva, con base en la media y la desviación estándar. Los resultados obtenidos revelaron que los componentes del proceso de la gestión del conocimiento están medianamente presentes en las gerencias estudiadas, siendo determinantes en el cumplimiento de los objetivos organizacionales; estableciendo la importancia de elaborar un diagnóstico interno a fin de establecer una valoración en base a las carencias y oportunidades de la organización.

Palabras clave: Gestión del conocimiento; proceso de gestión del conocimiento; organización industria petrolera; Venezuela

\section{ABSTRACT}

The purpose of the investigation was to describe the process of knowledge management in the management of aquatic operations of the oil industry in Zulia state. The type of research was descriptive, field, non-experimental transectional. The population was made up of 46 subjects among leaders, managers and supervisors. The survey was used, through a questionnaire, validated by the expert opinion. Reliability was determined by the Alpha Cronbach coefficient, whose value was 0.95 (very high reliability). Descriptive statistics were applied, based on the mean and standard deviation. The results obtained revealed that the components of the knowledge management process are moderately present in the management studied, being decisive in the fulfillment of the organizational objectives; establishing the importance of developing an internal diagnosis in order to establish an assessment based on the lacks and opportunities of the organization.

Key words: Knowledge management; knowledge management process; organization; oil industry, Venezuela 
INTRODUCCIÓN

El estudio del conocimiento humano ha sido objeto fundamental de la filosofía y la epistemología desde la época de los griegos, pero en los últimos años ha recobrado importancia y se le ha prestado más atención que antes. En el entorno actual de alta competitividad resulta clave la gestión del conocimiento, las organizaciones exitosas y competitivas son aquellas que se mantienen, la gestión del conocimiento representa una actividad de gestión y por lo tanto depende de la forma en que las personas dirijan a las empresas, y de las condiciones organizativas.

La gestión del conocimiento significa la evolución de la cultura empresarial, el cambio de estructuras organizativas, y está relacionada con la gestión del cambio, la transformación constante de rutinas de interacción y de procesos. Consecuentemente supone un dinamismo constante con estructuras organizativas más complejas, más matriciales, donde las personas tienen que interactuar con varias jerarquías simultáneas, con diferentes objetivos que pueden parecer a veces contradictorios.

En relación a la gestión del conocimiento; desde hace décadas la sociedad se ha venido preparando consciente o inconscientemente para lo que hoy en día conocemos como la sociedad del conocimiento, la sociedad en las organizaciones está expuesta a nueva exigencias, nuevos patrones de comportamiento, tanto de sus miembros como de sus usuarios, beneficiarios o clientes y nuevos paradigmas en el sistema económico y social, tanto nacional como internacional. Estos cambios han presionado hacia nuevas formas de administración de desarrollo institucional, que se caracterizan por el reconocimiento y auge de los llamados bienes intangibles y capital intelectual.

Existe una gran variedad de conceptos dados, por distintos investigadores, a la gestión del conocimiento; pero todos concuerdan en que la gestión del conocimiento es un proceso continuo de adquisición, distribución (en tiempo y forma a los que la necesiten) análisis de la información que se mueve en el entorno de la organización para hacer más inteligente a sus trabajadores (entiéndase como más creativos e innovadores). Se espera de esta forma ser más precisos en la toma decisiones, dar una respuesta más rápida a las necesidades del mercado, obtener un desarrollo sostenible ser más competitivos en este entorno tan turbulento, cambiante lleno de incertidumbre, la gestión del conocimiento acerca a entender el recurso humano como una capacidad desarrollable, susceptible de transformarse en una ventaja competitiva de la empresa.

En este orden de ideas, Dueñas (2014), define la gestión del conocimiento como la identificación de categorías de talento humano necesario para apoyar la estrategia empresarial global. Al evaluar el estado actual del know-how (saber hacer) y su capacidad de transformación de la base de habilidades y capacidades, se genera una nueva y poderosa base de competitividad, cubriendo la brecha de la cadena de valor en la organización.

La gestión del conocimiento ocupa indiscutiblemente un lugar prominente en la obtención y desarrollo de ventajas competitivas en las organizaciones. Pero, como se dijo en la década de los noventa un viejo adagio de gestión aquello que no se mide no se puede gestionar, es indispensable la existencia de modelos para el análisis de la 
medición, que operen sistemáticamente para articular las influencias recíprocas entre los elementos que conforman dicha actividad.

La generación de conocimientos demanda por parte de las empresas el desarrollo de capacidades para repensar, renovar, reordenar insumos, procesos y productos. Esto revela la existencia de una relación entre el conocimiento y la capacidad para innovar continuamente ya que ambos podrían ser considerados como factores de producción. Existe una correlación entre gestión del conocimiento y éxito empresarial, entre gestión del conocimiento con adaptabilidad de la empresa al entorno cambiante o desafiante, donde las amenazas se puedan convertir en oportunidades, esto evidencia la relación entre recursos, competencias y capacidades organizacionales con la innovación, el conocimiento asimismo con su gerencia propiamente dicha, para el logro de ventajas competitivas de las organizaciones.

Del mismo modo, la industria petrolera venezolana representada en este caso por la principal empresa Petróleos de Venezuela S.A. en lo sucesivo PDVSA no escapa del desarrollo global de la industrialización y en este caso específico las unidades de estudio estuvo constituida por las gerencias de Operaciones Acuáticas, se evidencia que existe un interés o la búsqueda de un continuo mejoramiento para el desarrollo de soluciones orientadas a incrementar la efectividad a través del intercambio de experiencias y conocimientos para generar las bases del conocimiento como valor para la organización logrando así una optimización continua de sus gestiones, con el propósito de elevar sus índices de confiabilidad operacional manteniendo sistemas tecnológicos vanguardistas $\mathrm{y}$ cumpliendo con los requerimientos del cliente.

Por lo tanto la gestión del conocimiento constituye una herramienta poderosa para promover la innovación, impulsando el mejoramiento continuo, desarrollando la creación de estrategias, métodos y tecnologías que permitan diseñar soluciones que realmente aporten valor a la empresa.

Finalmente el objetivo trazado en la investigación fue describir el proceso de gestión del conocimiento en las gerencias de operaciones acuáticas de la industria petrolera en el estado Zulia.

\section{Proceso de gestión del conocimiento}

El proceso de gestión del conocimiento debe entenderse como las fases necesarias para el desarrollo de soluciones orientadas a generar las bases del conocimiento de valor para la organización, es decir, representa la cadena de agregación de valor a cada una de las instancias de conocimiento existentes en la organización. Cabe destacar que el proceso de gestión del conocimiento se centra en el concepto de generación de valor asociado al negocio, el cual ayuda a descartar las instancias de conocimiento que sean no relevantes.

Por otro lado, López y Conde (2014) manifiestan que el proceso de gestión del conocimiento contempla sistemas que permiten el aumento del capital intelectual en forma significativa, con el objetivo de generar ventajas competitivas. Es la función que planifica, coordina y controla el flujo del conocimiento. De igual manera refieren, que es el proceso mediante el cual se adquiere, genera, almacena, comparte y utiliza el conocimiento, la información, ideas $y$ experiencias para mejorar la calidad en el cumplimiento y desarrollo de la misión de la 
organización. A continuación, se explican detalladamente cada uno de los componentes del proceso de gestión del conocimiento referenciando varios autores.

\section{a) Detección}

Las organizaciones deben pensar qué quieren lograr y el conocimiento necesario para ello, en tal sentido, dentro del proceso de detección de conocimiento, Pereira (2011), hace referencia a la necesidad de incluir un análisis enfocado o brechas para identificar el conocimiento que no se ha adquirido (sea tácito o explicito) a todos los niveles (estratégico, funcional, de procesos, personal, entre otros). Esta información es vital para la toma de decisiones; entonces para obtenerla se recomienda utilizar técnicas como los mapas del conocimiento, tormentas de ideas, retroalimentación con los clientes, experiencias de proyectos realizados, bases de datos para identificar a los aliados estratégicos, monitoreo de sitios web, entre otros.

Para Herranz y Vega (2011), al referirse sobre el proceso de gestión del conocimiento en una empresa u organización, el primer paso a dar, es la detección de los conocimientos, el cual residen en el seno de la misma así como de sus características o elementos identificativos. Este proceso constituye el núcleo del ciclo, y, representa su punto de arranque, es decir, es el elemento clave para poner en marcha cualquiera del resto de las fases del ciclo, ya que éstas girarán siempre sobre los conocimientos identificados. Es fundamental, por tanto, que una organización identifique cuáles son sus conocimientos, los explicite y formalice dicho proceso en un documento escrito, permitiendo su comunicación a todos los interesados dentro de la organización.
Adicionalmente, los autores Henrraz y Vega (2011), exponen que la detección de conocimientos se puede hacer de una u otra manera en función de cómo vayan a utilizarse los mismos posteriormente, para lo cual establecen dos (2) posibles usos:

- Detección de los conocimientos clave de la organización, entendiendo como conocimientos claves aquellos que sirven de apoyo a la empresa a fin de establecer una ventaja competitiva para el futuro. Dichos conocimientos determinaran las actividades a desarrollar, mantener $\mathrm{o}$ eliminar, las inversiones a realizar, estrategias, entre otros. En este caso, se debe responder a dos (2) cuestiones: (a) ¿Qué áreas de conocimiento son las más importantes en el negocio? y (b) ¿Qué ventajas de conocimientos serían más valorables en el negocio para mejorar su desarrollo y afianzamiento?

- Detección de los conocimientos necesarios para la creación de nuevos activos. En este caso, se trata de identificar conocimientos cara a su utilización para el diseño, producción, entre otros de nuevos productos $y$ servicios. Así, se detectan los conocimientos necesarios para prestar determinado servicio o, a la inversa, se definen los posibles servicios a generar en el futuro partiendo de los conocimientos ya existentes.

La detección, a juicio de Pavez (2009), es la fase de localizar modelos cognitivos y activos (pensamiento y acción) de valor para la organización, el cual radica en las personas. Son ellas, de acuerdo a sus capacidades cognitivas (modelos mentales, visión sistémica, $\mathrm{y}$ otros), quienes 
determinan las nuevas fuentes de conocimiento acción. Las fuentes de conocimiento pueden ser generadas tanto de forma interna (Investigación y desarrollo, proyectos, descubrimiento, entre otros); como externa (fuentes de información periódica, Internet, cursos de capacitación, libros).

Finalmente, Ponce y Falcón (2005), listan varios ejemplos de actividades con el propósito de alcanzar la detección e identificación en el contexto de la gestión del conocimiento: Revisión después de la acción, lecciones aprendidas, debriefing, balances de Know - How, portafolio del conocimiento, mapas del conocimiento, agente del conocimiento (Knowledge Brokers), tablero de comando (Balanced Scorecard), evaluación de patentes, entre otras.

\section{b) Organización}

Para Pavez (2009), la organización del conocimiento, es el proceso de almacenar de forma estructurada la representación explícita del modelo. Según el autor, este proceso se divide en generación, codificación y transferencia, definiéndolos en los siguientes términos:

- Generación: Es la creación de nuevas ideas, el reconocimiento de nuevos patrones, la síntesis de disciplinas separadas, y el desarrollo de nuevos procesos.

- Codificación: Es la representación del conocimiento para ser accedido y transferido por cualquier miembro de la organización a través de algún lenguaje de representación de codificación puede diferir de la representación de almacenamiento, dado que enfrentan objetivos diferentes: personas $y$ máquinas.
- Transferencia: Consiste en establecer tanto los medios de almacenamiento como de apertura que tendrá el conocimiento, ayudado por interfaces de acceso masivo (por ejemplo, la Internet o Intranet), además de establecer los criterios relativos a la seguridad como a su acceso. Además debe considerar aspectos tales como la barrera de tipo temporales (vencimiento), de distancias y sociales.

Por su parte, Herranz y Vega (2011), denominan a esta fase del proceso de gestión del conocimiento almacenaje, pues una vez detectado y seleccionado el conocimiento, el siguiente paso dentro del proceso es almacenarlo. Como se ha expuesto anteriormente, el conocimiento puede ser tácito o explícito, siendo el explícito transmisible a través del lenguaje formal, de una manera sistemática, al poder expresarse tanto en palabras como en números, mientras el tácito el constituido por la percepción subjetiva así como las emociones. Por tanto, al hablar de almacenar el conocimiento evidentemente se está refiriendo al conocimiento explícito, por ser susceptible de ser almacenado.

Los autores antes mencionados exponen que el almacenamiento del conocimiento hace referencia a dos procesos: (a) la externalización, que pretende la conversión de conocimiento tácito en explícito, traduciendo el conocimiento implícito de manera entendible por los demás y (b) la combinación, que supone la conversión de conocimiento explícito en explícito. Sin embargo, dado que una de las características del conocimiento es su intangibilidad, es necesario transformar el mismo en estructuras físicas, a fin de lograr su adecuado almacenamiento. Dicha 
transformación debe realizarse en un formato para almacenarlo, estructurarse, transmitirse, realimentarse, de manera sencilla $y$, sobre todo, sin recurrir a las personas que lo originaron.

Para ello, una vez que el conocimiento surge en la organización, se dispone de una gran variedad de medios para codificar el mismo: en papel, en formato multimedia, archivos de audio, entre otros. Sin embargo, el proceso de almacenamiento de conocimiento no consiste exclusivamente en su codificación, por ello es necesario interpretarlo, para darle un sentido coherente antes de hacerlo público. Así, el conocimiento debe ser almacenado de manera que pueda ser reutilizado por cualquiera, independientemente de quién haya originado dicho conocimiento.

Por otro lado, debe almacenarse en formatos que sean accesibles por parte de toda la organización. Es aquí donde las Tecnologías de la información y Comunicación (TIC) juegan un papel decisivo, facilitando estos procesos de almacenamiento. Es por ello, que, ante otras alternativas (bibliotecas, por ejemplo) se opta por llevar a cabo el proceso de almacenamiento del conocimiento utilizando dichas tecnologías.

La fase de filtración está íntimamente vinculada al almacenamiento del conocimiento, debe señalarse que, el conocimiento debe estructurarse de manera adecuada, de lo contrario, es imposible el acceso al mismo. Al respecto, Pavez (2009), sugiere la organización de la fuente, permitiendo así el acceso a través de consultas automatizadas entorno a motores de búsqueda. Éstas se basan en estructuras de accesos simples y complejos, tales como mapas de conocimiento o agentes inteligentes.
Por su parte, Herranz y Vega (2011), señalan que el conocimiento almacenado si no está estructurado de manera adecuada y lo convertirían en inútil. Por tanto, se trata de dos funciones que deben realizarse en paralelo (de hecho, en la mayoría de los casos se considera, en la práctica, como una sola fase del ciclo, la de almacenar y filtrar). A la hora de establecer cuál debe ser la estructura adecuada al conocimiento, es especialmente útil la distinción entre conocimiento percepcional, abstracto $\mathrm{y}$ experimental. Así, el conocimiento podría ser almacenado, de manera simple, con la siguiente estructura:

- Casos prácticos: recoge experiencias prácticas reales de la empresa que pudieran ser de utilidad cara al futuro, para resolver situaciones análogas 0 parecidas. Es el denominado conocimiento percepcional.

- Metodología: en este caso, se trata de deducir reglas y pautas en base al conocimiento poseído. Dicho de otra manera, se extrapolan casos particulares para obtener una regla general.

- Herramientas: en este caso, la experiencia se materializa en una serie de herramientas que le puedan resultar útiles a los empleados de la organización.

- Preguntas frecuentes: en este caso, se buscaría recoger un listado de preguntas frecuentemente realizadas y se le daría respuesta por parte del personal entrenado o experto en la organización.

- Fuentes de información: agrupa una relación de lugares donde se pudiera acceder a información adicional sobre la materia consultada.

- Foros: en este caso, se trataría de un lugar donde se comparte conocimiento de una manera interactiva. 
- Formación: recogería el acceso a oferta formativa que permitiera perfeccionar el conocimiento del empleado.

Evidentemente, se pueden generar otras estructuraciones. Finalmente, esta filtración debería hacerse de manera matricial. Esto quiere decir que en un eje se presenta esta clasificación realizada y en el otro, la relación de conocimientos identificados y reflejados en el mapa de conocimientos, de manera que cada persona puede acceder directamente al conocimiento de su interés en concreto, y desde ahí acceder a las distintas piezas donde se estructura el mismo.

\section{c) Presentación}

Una vez recogido y almacenado el conocimiento, llega el momento de hacerlo disponible en la organización. Se debe tener cuenta que el conocimiento es intrínsecamente social, porque no es posible reconocer los datos y la información como conocimiento al margen de algún tipo de contexto social. Por tanto, es necesario diseñar sistemas que permitan la presentación y difusión del conocimiento, esta presentación del conocimiento está unida indisolublemente con su compartición.

A criterio de Pavez (2009), los resultados obtenidos del proceso de filtrado deben ser presentados a personas $\mathrm{o}$ máquinas. En caso de personas, las interfaces deben estar diseñadas para abarcar el amplio rango de comprensión humana. En caso que la comunicación se desarrolle entre máquinas, las interfaces deben cumplir todas las condiciones propias de un protocolo o interfaz de comunicación.

Por su parte, Herranz y Vega (2011) plantean que una vez almacenado $\mathrm{y}$ estructurado el conocimiento en la organización, debe ser presentado y ponerse en circulación, las personas que accedan a él podrán completarlo, matizarlo y modificarlo con su propio conocimiento. Se estará, por tanto, intercambiando y compartiendo conocimiento, así como también creando uno nuevo. Por otro lado, al considerar la presentación del conocimiento, debe tenerse en cuenta dos vertientes: la presentación interna (entre los propios empleados, que es la variante habitualmente utilizada por la empresa) y la presentación externa (personas ajenas a la organización, lo cual, en la práctica, supone la comercialización del conocimiento), dichas variantes se explican detalladamente a continuación:

El reto, por tanto, es retener al personal, mantener y mejorar dichos sistemas $\mathrm{y}$ continuar generando ese conocimiento (es decir, gestionar el conocimiento de forma continua), de manera que la organización siempre este un paso por delante de los competidores. Por otro lado, este enfoque siempre es especialmente válido si se hace referencia a organizaciones que no se encuentren en un mercado competitivo y su finalidad sea otra, tales como: organizaciones gubernamentales, universidades, fundaciones o empresas en régimen de monopolio. También, en un entorno cada vez más globalizado, experiencias en un sector pueden resultar de interés en otros, lo cual facilita la exportación del conocimiento.

\section{d) Uso}

El conocimiento añade valor solamente cuando se utiliza en la organización; el exceso de este no se utiliza a plenitud. En tal sentido, Pereira (2011), plantea que el uso del conocimiento determina las necesidades de la organización a este respecto, y debe servir como referencia para la creación, almacenamiento y las formas de compartir 
conocimiento. Es posible que se descubran otras brechas cuando se aplica el conocimiento, por ello el proceso global de gestión del conocimiento debe realizarse de forma continua para asegurar que se integra a la iniciativa gestión del conocimiento.

Por su parte, Pavez (2009), expone que el uso del conocimiento reside en el acto de aplicarlo al problema objeto de resolver. De acuerdo con esta acción es posible evaluar la utilidad de la fuente de conocimiento a través de una actividad de retroalimentación. Cabe destacar que el proceso de gestión del conocimiento propuesto se centra en la generación del valor, por lo que el centro de dirección del proceso es el negocio.

Finalmente, Ponce y Falcón (2005), listan varios ejemplos de algunas de las acciones que las organizaciones pueden implementar con la finalidad de incrementar el uso del conocimiento, siendo estas: Conferencias internas, videoconferencias, foros de discusión, intranet, publicaciones empresariales sobre empleados, grupos de noticias, circulares, encuentros de conocimiento (internos), congresos, seminarios, entre otros.

\section{e) Mantenimiento}

Una vez creado, almacenado, estructurado y distribuido, el ciclo de vida del conocimiento no se termina, ya que a juicio de Ponce y Falcón (2005), el conocimiento también debe evolucionar, para mantenerse al día de los cambios que se producen en el entorno, de lo contrario, perderá su valor. Esto requiere de programas específicos cuya finalidad sea aprovechar la experiencia acumulada, estimular los procesos creativos y/o de I+D, rastrear el mercado en busca de innovaciones, nuevos productos y/o servicios. Esta tarea de mantenimiento del conocimiento debe ser asumida por toda la organización. Una vez en marcha todo el ciclo, el mantenimiento debe ser consecuencia natural, fruto de la creación de nuevo conocimiento, de la distribución y compartición del mismo (Fontalvo, et al. 2011).

En cuanto al mantenimiento de los sistemas y las herramientas diseñadas e implantadas, debería ser responsabilidad directa de las unidades funcionales directamente e involucradas con los mismos. Por ejemplo, García y Arregui (2009), exponen que al diseñar un sistema de apoyo al personal de atención al cliente este debe recopilar toda la experiencia y el know-how existente en la organización, donde el mantenimiento de este sistema es responsabilidad de la dirección comercial.

\section{f) Contabilización}

Los estados financieros de la empresa, aun siendo fiables, han dejado de ser reales, ya que no reflejan el valor de la empresa. Esto como consecuencia que los activos intangibles (el conocimiento) constituye una parte fundamental de dicho valor. Asimismo, las inversiones en dichos intangibles son vitales para el mantenimiento de las ventajas competitivas. Por tanto, se hace necesario desarrollar indicadores que permitan medir dichos activos intangibles a fin de mejorar la gestión, así como valorar correctamente la empresa. Este proceso es denominado por Pereira (2011), como contabilización o medición del conocimiento. El mismo supone valorar la contribución del conocimiento existente en la empresa al valor de la misma y constituye el cierre del ciclo; este paso permite medir la efectividad de gestionar los activos intangibles. 
Sin embargo, no es una tarea fácil, ya que esta es una información de tipo subjetivo, que por naturaleza no puede codificarse de manera matemática. En este sentido, García y Arregui (2009), plantean que se han puesto en marcha varias iniciativas con el fin de establecer un sistema que objetivase lo mejor posible dicha medición, mediante el establecimiento de un sistema de indicadores que permitan medir, de manera universal, el valor de los intangibles de una organización.

Adicionalmente, García y Arregui (2009), hacen referencia a otros enfoques para la contabilización del conocimiento, siendo estos: Cuadro de Mando Integral, desarrollado por Kaplan y Norton, el monitor de activos intangibles (Intangible Assets Monitor), del sueco Sveiby, el modelo de dirección estratégica por competencias, desarrollado por el Grupo de Trabajo de Intelect, el modelo Technology Broker de Annie Brooking y el modelo MERITUM, desarrollado entre varias universidades europeas. Sobre el proceso descrito anteriormente, es posible desarrollar el concepto de proyecto de gestión del conocimiento, el cual tiene como objetivo generar las instancias que reflejen de manera práctica cada una de las etapas del proceso.

Como puede observarse, la gestión del conocimiento está asociada a los procesos y procedimientos de actividad de cada organización, al capital intelectual (recursos humanos) y a la documentación e información. Por tanto, gestionar el conocimiento es, en gran medida gracias a la contribución del capital intelectual con que cuenta la organización.

\section{MÉTODO}

La investigación correspondió al estadio descriptivo, con un diseño de campo, transeccional, no experimental. Se utilizó la encuesta como técnica y el instrumento fue un cuestionario, validado por el juicio de expertos, cuya confiabilidad se determinó mediante el Coeficiente de Alpha Cronbach, donde se obtuvo un valor de 0,95 indicando muy alta confiabilidad. Con relación al análisis de los datos se realizó mediante la estadística descriptiva, con base en la media y la desviación estándar. A continuación se presenta los cuadros 1 y 2 , donde respectivamente se muestran las categorías de análisis para la interpretación de la media e interpretación de la desviación estándar.

Cuadro 1. Categorías de análisis para la interpretación de la media

\begin{tabular}{cllll}
\hline Rango & Intervalo & Alternativas & Categoría & Descripción \\
\hline 1 & $1-1,80$ & Nunca & Ausente & $\begin{array}{l}\text { La respuesta se ubica en el rango } \\
\text { muy negativo. }\end{array}$ \\
2 & $1,81-2,60$ & Casi Nunca & Poco presente & $\begin{array}{l}\text { La respuesta se ubica en el rango } \\
\text { negativo. } \\
\text { La respuesta se ubica en el rango } \\
\text { positivo o negativo. }\end{array}$ \\
4 & $2,61-3,40$ & Algunas veces & $\begin{array}{l}\text { Mediantemente } \\
\text { presente }\end{array}$ & $\begin{array}{l}\text { La respuesta se ubica en el rango } \\
\text { positivo. }\end{array}$ \\
5 & $4,41-4,20$ & Casi siempre & Presente & $\begin{array}{l}\text { La respuesta se ubica en el rango } \\
\text { muy positivo }\end{array}$
\end{tabular}


Cuadro 2. Análisis para la interpretación de la desviación estándar

\begin{tabular}{|c|c|c|}
\hline Intervalo & Categoría & Descripción \\
\hline $1,61-2,00$ & Muy alta & $\begin{array}{l}\text { Indica una muy alta dispersión de las respuestas y, una } \\
\text { muy baja confiabilidad de las mismas. }\end{array}$ \\
\hline $1.21-1,60$ & Alta & $\begin{array}{l}\text { Indica una alta dispersión de las respuestas y, una baja } \\
\text { confiabilidad de las mismas. }\end{array}$ \\
\hline $0,81-1,20$ & Moderada & $\begin{array}{l}\text { Indica una moderada dispersión de las respuestas y, una } \\
\text { moderada confiabilidad de las mismas. }\end{array}$ \\
\hline $0.41-0,80$ & Baja & $\begin{array}{l}\text { Indica una baja dispersión de las respuestas y, una alta } \\
\text { confiabilidad de las mismas. }\end{array}$ \\
\hline $0-0.40$ & Muy baja & $\begin{array}{l}\text { Indica una muy baja dispersión de las respuestas y, una } \\
\text { muy alta confiabilidad de las mismas }\end{array}$ \\
\hline
\end{tabular}

\section{RESULTADOS Y DISCUSIÓN}

Tabla 1. Procesos de la gestión del conocimiento

\begin{tabular}{cccc}
\hline Indicadores & $\overline{\mathrm{X}}$ & $\mathbf{S}$ & Categoría de Interpretación \\
\hline Detección & 2,96 & 0,70 & Medianamente presente/ Baja dispersión \\
Organización & 3,05 & 0,59 & Medianamente presente/ Baja dispersión \\
Presentación & 3,28 & 0,92 & Medianamente presente/ Moderada dispersión \\
Uso & 2,72 & 0,76 & Medianamente presente/ Baja dispersión \\
Mantenimiento & 2,85 & 0,69 & Medianamente presente/ Baja dispersión \\
Contabilización & 3,04 & 0,66 & Medianamente presente/ Baja dispersión \\
Resumen & $\mathbf{2 , 9 8}$ & $\mathbf{0 , 7 2}$ & Medianamente presente/ Baja dispersión \\
\hline
\end{tabular}

En relación al objetivo específico el proceso de gestión del conocimiento en las gerencias de operaciones acuáticas de la industria petrolera en el estado Zulia. En la tabla 1 se reflejan los resultados estadísticos obtenidos con respecto a los indicadores detección, organización, presentación, uso mantenimiento y contabilización, lo cual permitió analizar los resultados de la dimensión procesos de la gestión del conocimiento.

Sobre el indicador detección, se observó una media de 2,96 (medianamente presente) con desviación 0,70 (baja dispersión), se puede inferir que los sujetos encuestados consideran que algunas veces se determinan los conocimientos claves que le servirán de apoyo a la hora de establecer ventajas competitivas. Así, Herranz y Vega (2011), expresan que los conocimientos claves sobre los cuales la organización se apoyará, servirá de base para desarrollar las estrategias para establecer las ventajas competitivas.

Para el indicador organización, la media es de 3,05 (medianamente presente) y desviación 0, 59 (muy baja dispersión), denota que el personal encuestado está claro que algunas veces se codifica el conocimiento para que pueda ser accedido y transferido por cualquier miembro de la 
organización, Al respecto, Herranz y Vega (2011), señalan que una vez que el conocimiento surge en la organización existe una gran variedad de métodos para codificarlo y/o almacenarlo de manera tal que pueda ser utilizado y de acceso por cualquier integrante de la organización.

En relación al indicador presentación, la media registrada fue de 3,28 (medianamente presente) con desviación de 0, 92 (moderada dispersión), es indicativo de la existencia de sistemas de información organizacional integrados en una red interna (intranet) a través del cual el talento humano accede al conocimiento de su interés. Herranz y Vega (2011), establecen, el conocimiento se pone a disposición de los empleados de distintas formas diferentes y él mismo puede ser distribuido mediante la utilización de sistemas integrados o interna (intranet) de manera tal que pueda ser utilizado para la toma de decisiones.

Analizando el indicador uso, el valor para la media 2,72 (medianamente presente) con desviación 0,76 (baja dispersión), es decir los encuestados consideran que la organización, solo algunas veces la experiencia previa es utilizada para la creación de nuevos conocimientos y en algunos casos se aplica el conocimiento organizacional para la generación de valor agregado en los procesos que se ejecutan.En tal sentido, Pereira (2011), plantea que las necesidades de la organización están determinadas por el uso del conocimiento, por cuanto este añade valor y debe servir como referencia para la creación a este respecto, y debe servir como referencia para la creación, almacenamiento y las formas de compartir conocimiento.

En cuanto al indicador mantenimiento, el valor para la media fue de 2,85 (medianamente presente) y desviación 0,69 (baja dispersión), por tanto se infiere que para los sujetos encuestados solo algunas veces se aprovecha la experiencia acumulada con la finalidad de mantener vigente el conocimiento organizacional. Ponce y Falcón (2005) postulan, que el ciclo de vida del conocimiento no se termina y que este para no perder su valor debe evolucionar y mantenerse actualizado.

De igual forma para el indicador contabilización, la media registrada fue de 3,04 (medianamente presente) con desviación 0,66 (baja dispersión), se infiere que para los encuestados solo algunas veces se estimulan procesos creativos en el talento humano dirigidos a actualizar el conocimiento organizacional, como contabilización o medición del conocimiento. Pereira (2011), postula que la contabilización del conocimiento; es un indicador que permite medir la efectividad con la que se han gestionado los activos intangibles, y constituye el cierre del ciclo. Es posible afirmar que en esta dimensión existen debilidades por cuanto se supone valorar la contribución del conocimiento existente a la organización al valor de la misma ya que constituye el cierre del ciclo, $y$ permite medir la efectividad con la que se han gestionado los activos intangibles.

Resumiendo en lo referente a los procesos de la gestión del conocimiento, se aprecia una media de 2,98 con desviación 0,72 (baja dispersión). Esto demuestra que los procesos de la gestión del conocimiento son manejados de tal forma que afecten positivamente el desempeño de la organización, sin embargo se aprecian aspectos que se convierten en debilidades para la organización, y deben ser atendidas en función de un mejoramiento continuo. Cabe destacar que el proceso de gestión del conocimiento se centra en el concepto de 
generación de valor asociado al negocio, el cual ayuda a descartar las instancias de conocimiento que sean no relevantes.

En el mismo contexto para efectos de efectos de la investigación, el proceso de gestión del conocimiento representa la cadena de agregación de valor en la organización y se centra en el concepto de generación de valor asociado al negocio, necesaria para el desarrollo de soluciones orientadas a generar las bases del conocimiento para la organización.

\section{CONCLUSIONES}

La gestión del conocimiento no es solo un proceso de almacenamiento y acceso a la información, sino un proceso dirigido a generar valor para la organización, en las que se presentan diferentes facetas, tales como la creación de una cultura adecuada, identificación de necesidades de formación, captura, asimilación, y utilización de los conocimientos lo anteriormente señalado, indica que la combinación sinérgica de elementos como productos, procesos, mercados, proveedores, clientes, empleados, así como también la manera de desarrollarlo adecuadamente para diferenciarse del resto, le permite a la empresa ser más competitiva, claro está que el factor clave para el éxito es el capital humano.

Una vez finalizada la investigación y en función de los resultados obtenidos se pueden establecer las siguientes conclusiones:

Los indicadores detección y organización, aun y cuando se encuentran medianamente presente se observan importantes debilidades que deben ser corregidas ya que no se evidencia estrategias definidas que sirvan de apoyo para la creación de ventajas competitivas.
Así mismo para los indicadores presentación y uso aun y cuando es positivo, existen debilidades en los sistemas informáticos existentes, y finalmente en términos generales para el indicador mantenimiento, se aprovecha algunas veces la experiencia acumulada, se debe ir en la búsqueda de programas más específicos en la búsqueda de innovaciones a fin de aprovechar la experiencia acumulada y estimular los procesos creativos.

Referente al indicador contabilización, este indicador representa una debilidad y debe ser atendido por cuanto representa el cierre del ciclo y de deben realizarse indicadores de gestión, esto permite tener una idea de medir la efectividad con la que se gestionan los activos intangibles.

Finalmente se concluye que en las gerencias de operaciones acuáticas de la industria petrolera en el estado Zulia, se debe considerar y gestionar el conocimiento potenciarlo, difundirlo, para utilidad de los integrantes a diferentes niveles de la empresa, se deben hacer los esfuerzos por definir las mejoras a fin incrementar las capacidades de aprendizaje de los individuos.

\section{REFERENCIAS}

Dueñas, H. (2014). Gestión del conocimiento y capital Intelectual. Recuperado de: http://www.ceipa.edu.co/lupa/index.ph p/lupa/article/view/56/106

Fontalvo, T., Quejada, R., y Puello P. (2011). La gestión del conocimiento $\mathrm{y}$ los procesos de mejoramiento. Dimensión Empresarial, 9(1), 80-87

García, J; y Arregui, T. (2009). Gestión del conocimiento y empresa. Una aproximación a la realidad española. Barcelona, España: Colección EOI 
Herranz, A; y Vega, R. (2011). La gestión del conocimiento como vía de consolidación y crecimiento en la empresa española. Escuela de Negocios. Madrid, España: Editorial EOI

López, J; Conde, A. (2014).Gestión del conocimiento en organizaciones empresariales. Facultad de Ciencias de la Educación de Granada. Departamento de Didáctica y Organización Escolar. España: Grupo EDO

Pavez, A. (2009). Modelo de implantación de Gestión del Conocimiento y Tecnologías de Información para la Generación de
Ventajas Competitivas. Madrid, España: Editorial Mc Graw Hill

Pereira, H. (2011). Implementación de la gestión del conocimiento en la empresa. Revista CEGESTI. No. 135. Recuperado de:

http://www.cegesti.org/exitoempresari al/publicaciones/publicacion_135_3101 11_es.pdf

Ponce, A. y Falcón, P. (2005).Metodología para realizar la Introducción de la Gestión del Conocimiento en las Organizaciones. Centro de Información y Gestión Tecnológica Cienfuegos 